\title{
Papers
}

\section{Is voice therapy an effective treatment for dysphonia? A randomised controlled trial}

\author{
Kenneth MacKenzie, Audrey Millar, Janet A Wilson, Cameron Sellars, Ian J Deary
}

\begin{abstract}
Objectives To assess the overall efficacy of voice therapy for dysphonia.

Design Single blind randomised controlled trial. Setting Outpatient clinic in a teaching hospital. Participants 204 outpatients aged 17-87 with a primary symptom of persistent hoarseness for at least two months.

Interventions After baseline assessments, patients were randomised to six weeks of either voice therapy or no treatment. Assessments were repeated at six weeks on the $145(71 \%)$ patients who continued to this stage and at 12-14 weeks on the $133(65 \%)$ patients who completed the study. The assessments at the three time points for the 70 patients who completed treatment and the 63 patients in the group given no treatment were compared.

Main outcome measures Ratings of laryngeal features, Buffalo voice profile, amplitude and pitch perturbation, voice profile questionnaire, hospital anxiety and depression scale, clinical interview schedule, SF-36.

Results Voice therapy improved voice quality as assessed by rating by patients $(\mathrm{P}=0.001)$ and rating by observer $(\mathrm{P}<0.001)$. The treatment effects for these two outcomes were 4.1 (95\% confidence interval 1.7 to 6.6$)$ points and 0.82 (0.50 to 1.13$)$ points.

Amplitude perturbation showed improvement at six weeks $(\mathrm{P}=0.005)$ but not on completion of the study. Patients with dysphonia had appreciable psychological distress and lower quality of life than controls, but voice therapy had no significant impact on either of these variables.

Conclusion Voice therapy is effective in improving voice quality as assessed by self rated and observer rated methods.
\end{abstract}

\section{Introduction}

Many patients have transient, self limiting changes in voice, but those who have been hoarse for more than three weeks need specialist assessment to exclude underlying laryngopharyngeal pathology. Once conditions that need surgery have been excluded, patients are usually referred to a speech and language therapist for voice therapy. Up to 40000 patients with dysphonia are referred for voice therapy annually in the United Kingdom. ${ }^{1}$ At the time of referral, many patients with vocal dysfunction have entered a vicious cycle in which psychological factors exacerbate voice pathology and poor voice quality adversely affects psychological wellbeing. ${ }^{2-9}$ The relation between these factors is complex, and the relative influence of each factor varies from individual to individual.

No study has yet examined the overall effectiveness of voice therapy for dysphonia in terms of either changes in voice quality or changes in psychological distress or laryngoscopic findings. We aimed to examine the efficacy of voice therapy in patients with dysphonia and to identify those patients for whom voice therapy might be most beneficial.

\section{Participants and methods}

We recruited consecutive outpatients attending the department of otorhinolaryngology and head and neck surgery of Glasgow Royal Infirmary with a primary complaint of dysphonia (hoarseness) present for a minimum of two months and without any relevant organic pathology (for example, polyp, papilloma, tumour, vocal cord palsy) or need for surgery.

The inclusion criteria were age greater than 16 years, motivation to resolve the voice problem, and willingness to enter into regular voice therapy sessions. The exclusion criteria were previously treated dysphonia, neurological disease, or upper aerodigestive tract malignancy; marked hearing impairment; acid reflux; multiple medical complaints; professional voice user requiring urgent intervention; puberphonia; and transsexual conflict.

The 204 patients (51 men, 153 women) who gave informed consent for inclusion were new referrals typical of patients referred for voice therapy. At entry to the study, 100 patients were randomised to voice therapy and 104 to no treatment. By completion of the study 12-14 weeks later, about a third of participants had dropped out or been excluded, leaving 70 patients in the treatment group and 63 patients in the observation group (figure). This attrition was mostly a result of patients defaulting. In addition to failure to reattend, some patients failed to complete all self report questionnaires, notably where these had to be returned by post, despite the issuing of prepaid envelopes. Another subgroup omitted a number of items within questionnaires. The voice therapy and no treatment groups were not significantly different in terms of \begin{tabular}{ll} 
Editorial by Carding & \\
\hline &
\end{tabular} 


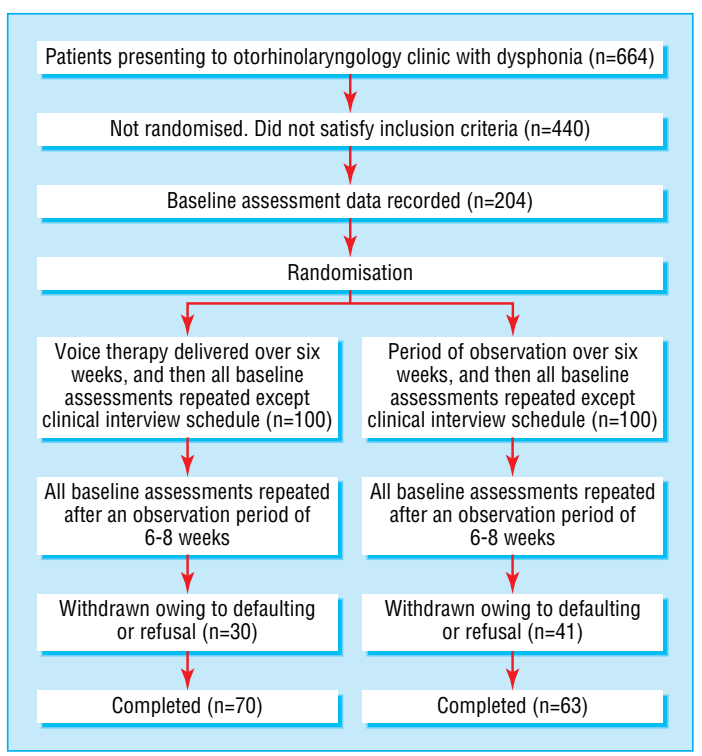

Flow chart of study

either rate of attrition (30\% in the therapy group, 39\% in the no treatment group) or characteristics of patients who dropped out (sociodemographic variables or baseline voice or psychological variables). There is thus no evidence that the attrition will have introduced bias. Analysis of each outcome was conducted only on patients with complete data.

\section{Measures}

Pathophysiology - An otolaryngologist (KMacK) used a flexible nasolaryngoscope (Olympus ENF 3) to assess four features-nodule formation, laryngitis, glottic escape, and hyperfunction of the laryngeal musculature-on a four point (0-3) rating scale. Although stroboscopic findings may facilitate the diagnosis of subtle laryngeal dysfunction, we decided not to use our stroboscope because we wanted the study to reflect routine clinical practice throughout the United Kingdom.

Voice quality-A digital tape recording of the patient's reading of the phonetically balanced "rainbow" passage (a standard paragraph used in voice assessment) was analysed by a speech and language therapist blind to treatment group. ${ }^{10}$ Ten aspects of voice, including laryngeal tone, pitch, and resonance, were rated $0-5$ on the Buffalo voice profile scale, ${ }^{11}$ of which the "overall rating" item was chosen as the key observer rated variable. The same therapist also extracted two key objective measures of voice quality"jitter" (pitch perturbation) and "shimmer" (amplitude perturbation)-by using the Computerised Speech Laboratory (model 4300B; Kay Elemetrics Corp, NJ). The higher the score on these two variables the more dysphonic the voice. Patients rated their own voice quality using the validated vocal performance questionnaire, ${ }^{12}$ with five point scoring on 12 items (1-5, 5 =worst). The total voice profile questionnaire score was the key self reported outcome measure.

Psychological measures-The hospital anxiety and depression scale is a self completed questionnaire which assesses recent anxiety and depression and provides an overall score for each. ${ }^{13}$ The revised clinical interview schedule was conducted by a trained psychologist blind to the treatment group. ${ }^{14}$ The interviewer rated 14 aspects of non-psychotic psychiatric disturbance. The clinical interview schedule's overall distress score and the hospital anxiety and depression scale's anxiety score were the key outcome measures of psychological distress.

Quality of life was assessed by the SF-36, an eight dimension, extensively validated assessment of general health status. ${ }^{15}$

\section{Intervention}

Baseline data were recorded after eligibility had been assessed and consent had been obtained by the laryngologist. The participants were then seen by one speech and language therapist (CS), who obtained a number for random allocation of the participant to either a course of voice therapy or a period of observation. Computer generated random numbers produced

\section{Treatment protocol}

The voice therapy group initially comprised 100 patients; eight patients were excluded from the study before therapy. These exclusions occurred for a range of reasons, most of which (for example, dementia) only emerged at the follow up appointment. Two patients withdrew from the study after the first visit. In addition to the eight patients who were excluded after allocation to the therapy group (two of whom withdrew), eight patients failed to attend the first therapy appointment. A further 10 patients defaulted during therapy. This meant that 74 patients completed a course of voice therapy, 70 of whom had complete data sets.

Participants entering the treatment group of the study were given an appointment at which a routine voice history was taken. A recording of the "rainbow" passage was taken for possible reference in therapy. In line with current practice, ${ }^{16}$ and depending on clinical circumstances, a number of patients (11 out of the 74 who completed treatment) received advice on good vocal hygiene and optimal voice production and did not proceed to a fuller programme of voice therapy, although this option remained open to them until attendance for the second visit. The remainder of the patients (63) underwent voice therapy for up to six sessions (eight sessions in one case). The treatment sessions lasted 45-60 minutes. The number of sessions needed and the type of treatment carried out were determined heuristically, depending partly on the nature of the symptoms and partly on patients' priorities. ${ }^{16}$ Treatment could be indirect-for example, involving discussion of issues of vocal hygiene or of lifestyle impinging on voice production. Equally, patients could be invited to practise techniques related to their vocal symptoms-for example, improving breath support for vocal production or altering vocal onset in favour of "softer" vocal attack. Because of the limited possibilities for contact with patients, therapy favouring attention to vocal symptoms predominated, but issues were incorporated relating to possible underlying psychological distress. Indeed, where these were clearly the focus of concern to the patient, a counselling approach was adopted.

The programme of voice therapy ended by mutual agreement between the treating therapist and the individual patient. Completion of therapy did not imply complete recovery at that time but rather a recognition that good vocal practice needed ongoing attention over a sustained period beyond the contact limits set for this project. 
in a restricted randomisation form were supplied by an independent worker in a separate department. All voice therapy was delivered by CS according to a protocol (see box) derived from a review of the type, form, and frequency of voice therapy used by a substantial sample of speech and language therapists in the United Kingdom. ${ }^{16}$ CS was not involved in the collection, storage, or analysis of outcome data in any way. Equally, the details of the treatment of this group were at no time communicated to any member of the research assessment team involved in collecting outcome data. After six weeks of therapy or observation, data on pathophysiology, voice quality, psychological status, and quality of life (with the exception of the clinical interview schedule) were recorded. After a further 6-8 weeks, all measurements were repeated, and the clinical interview schedule was conducted.

Before the study, the intended number of patients to be recruited in the treatment and non-treatment groups was determined by assuming a medium effect size of treatment ( $0.5 \mathrm{SD}$ units). This is a conservative effect size compared with that indicated in the available literature. Also, greater than $90 \%$ power was sought with $\alpha$ set at 0.05 (two tailed). The target chosen was 100 patients in each group, which offered $94 \%$ power. At the end of the study we had data from 70 and 63 people in the two groups; with the same assumptions, this offered $81 \%$ power.

\section{Statistical analysis}

Statistical analyses compared the mean difference in the outcome variables between the groups with and without treatment. We conducted separate analyses at the end of treatment and at the later follow up. Each analysis took into account the baseline (entry to study) score for each of the outcome variables. We thus used an analysis of covariance procedure with group (treatment versus no treatment) as a between patients variable; we used people's baseline scores on the particular variable being compared as covariates. We used baseline values as the covariates for both the end of treatment and follow up analyses.

Assessment of the effect of voice therapy on pathophysiological outcomes needed a categorical approach. We subtracted ratings for each pathophysiological feature for each patient at the end of treatment (visit 2) from those at baseline (visit 1); we also subtracted ratings for each feature at the end of follow up (visit 3) from those at visit 1 . We then assigned patients to a category $(0,1$, or 2$)$ according to whether they had improved, deteriorated, or stayed the same. We performed a series of $\chi^{2}$ intergroup comparisons.

We calculated treatment effects as mean differences at the relevant outcome (visits 2 and 3) controlled for baseline scores in the respective measure. We used general linear modelling (analysis of covariance) in SPSS $9 / 10$ to perform the analysis.

\section{Results}

As expected, most patients in both groups were women; the groups were closely matched for age (table 1). Laryngeal features at study entry were similar in the intervention and control patients (either in the 204 patients originally recruited or in the 145 with repeat
Table 1 Laryngoscopic data for treatment and no treatment groups at baseline and first follow up (only those who attended at first follow up are included at baseline). Values are numbers (percentage) of patients unless otherwise stated

\begin{tabular}{|c|c|c|c|c|}
\hline & \multicolumn{2}{|c|}{ No treatment $(n=72)$} & \multicolumn{2}{|c|}{ Treatment $(n=73)$} \\
\hline & Baseline & Follow up & Baseline & Follow up \\
\hline No (\%) female & $50(69)$ & & $56(77)$ & \\
\hline Mean (SD) age (years) & $52.0(13.2)$ & & $50.6(13.8)$ & \\
\hline \multicolumn{5}{|l|}{ Nodules: } \\
\hline 0 & $59(82)$ & $62(86)$ & $64(88)$ & $68(93)$ \\
\hline 1 & $9(13)$ & $8(11)$ & $7(10)$ & $3(4)$ \\
\hline 2 & $3(4)$ & $2(3)$ & $2(3)$ & $1(1)$ \\
\hline 3 & $1(1)$ & 0 & 0 & $1(1)$ \\
\hline \multicolumn{5}{|l|}{ Laryngitis: } \\
\hline 0 & $46(64)$ & $46(64)$ & $42(58)$ & $43(59)$ \\
\hline 1 & $20(28)$ & $19(26)$ & $19(26)$ & $22(30)$ \\
\hline 2 & $6(8)$ & $7(10)$ & $12(16)$ & $8(11)$ \\
\hline 3 & 0 & 0 & 0 & 0 \\
\hline \multicolumn{5}{|l|}{ Glottic escape: } \\
\hline 0 & $58(81)$ & $62(86)$ & $54(74)$ & $61(84)$ \\
\hline 1 & $13(18)$ & $9(13)$ & $16(22)$ & $10(14)$ \\
\hline 2 & $1(1)$ & $1(1)$ & $3(4)$ & $2(3)$ \\
\hline 3 & 0 & 0 & 0 & 0 \\
\hline \multicolumn{5}{|l|}{ Hyperfunction: } \\
\hline 0 & $63(88)$ & $65(90)$ & $60(82)$ & $68(93)$ \\
\hline 1 & $5(7)$ & $4(6)$ & $10(14)$ & $4(5)$ \\
\hline 2 & $3(4)$ & $3(4)$ & $3(4)$ & $1(1)$ \\
\hline 3 & $1(1)$ & 0 & 0 & 0 \\
\hline
\end{tabular}

laryngoscopy at six weeks). Grade 2-3 (moderate to severe) scores were uncommon for all of the four features, and only minimal resolution of the abnormalities occurred between the two time points (table 1).

The groups were well matched at entry to the study for subjective and objective voice variables (table 2). The treatment and no treatment groups differed at baseline only on the hospital anxiety and depression scale anxiety scores, which were significantly higher in the control group $(t=2.67, \mathrm{P}=0.008)$.

This difference between the treatment and no treatment groups was evident in the original 204 randomised recruits and in the 133 patients who completed all three phases of the study. Both the treatment and no treatment groups had high baseline scores for anxiety on the hospital anxiety and depression scale compared with healthy controls from the scale's normative reference data, though not necessarily with other otorhinolaryngology patients. ${ }^{89}$ The baseline SF-36 quality of life scores reflect a severely impaired quality of life in both the treatment and no treatment groups compared with other groups of patients. ${ }^{15}$

\section{Effectiveness of voice therapy}

By the end of treatment voice therapy significantly improved self rated quality of voice as measured by the voice profile questionnaire and the measurement of amplitude perturbation or "shimmer" by the Computerised Speech Laboratory. At follow up the patients in the treatment group had significantly lower scores than those in the no treatment group on the Buffalo overall rating and the voice profile questionnaire total score. Treatment effects (points) and 95\% confidence intervals were calculated for each of the outcome variables at both completion of treatment and completion of follow up (tables 2 and 3). All participants with data at baseline and follow up were included. For the voice profile questionnaire the effect was 4.1 points (effect size $0.54 \mathrm{SD}$ ). For the Buffalo scale the effect was 0.82 
Table 2 Mean (SD) scores for key voice and psychological variables at each visit for treatment and no treatment groups (patients with complete data)

\begin{tabular}{|c|c|c|c|c|c|c|c|c|}
\hline \multirow[b]{2}{*}{ Test or questionnaire } & \multicolumn{3}{|c|}{ Treatment } & \multicolumn{3}{|c|}{ No treatment } & \multirow[b]{2}{*}{$F$ value } & \multirow[b]{2}{*}{$\begin{array}{l}\text { Treatment effect } \\
\text { (points }(95 \% \mathrm{CI}) \text { ) }\end{array}$} \\
\hline & $\begin{array}{c}\text { No of } \\
\text { patients }\end{array}$ & $\begin{array}{c}\text { Visit } 1 \\
\text { (baseline) }\end{array}$ & Visit 2 or 3 & $\begin{array}{c}\text { No of } \\
\text { patients }\end{array}$ & $\begin{array}{c}\text { Visit } 1 \\
\text { (baseline) }\end{array}$ & Visit 2 or 3 & & \\
\hline \multicolumn{9}{|c|}{ At completion of treatment (visit 2) } \\
\hline \multicolumn{9}{|c|}{ Psychological distress: } \\
\hline HADS anxiety & 67 & $6.3(4.0)$ & $5.9(4.5)$ & 63 & $8.1(4.3)$ & $7.7(3.9)$ & $0.71(\mathrm{P}=0.402)$ & $0.39(-0.53$ to 1.31$)$ \\
\hline HADS depression & 67 & $3.7(3.5)$ & $3.6(3.7)$ & 63 & $4.2(3.3)$ & $4.2(3.1)$ & $0.38(\mathrm{P}=0.540)$ & $0.25(-0.55$ to 1.05$)$ \\
\hline CIS total score & 67 & $7.0(6.9)$ & & 62 & $8.6(8.2)$ & & & \\
\hline \multicolumn{9}{|l|}{ Voice: } \\
\hline Buffalo overall rating* & 74 & $2.9(1.1)$ & $2.3(0.93)$ & 69 & $2.9(1.0)$ & $2.5(0.93)$ & $2.33(P=0.130)$ & $0.21(-0.01$ to 0.49$)$ \\
\hline Pitch perturbation† & 67 & $3.2(2.4)$ & $2.8(1.5)$ & 57 & $2.4(1.4)$ & $2.8(1.5)$ & $0.97(P=0.326)$ & $0.25(-0.25$ to 0.74$)$ \\
\hline Amplitude perturbation $\dagger$ & 67 & $6.0(4.2)$ & $4.5(2.6)$ & 57 & $5.0(3.3)$ & $5.7(3.8)$ & $8.03(P=0.005)$ & $1.6(0.47$ to 2.63$)$ \\
\hline VPQ total & 67 & $27.6(8.0)$ & $22.4(5.8)$ & 65 & $27.9(7.5)$ & $25.4(8.1)$ & $6.12(P=0.015)$ & 2.6 (0.51 to 4.63$)$ \\
\hline \multicolumn{9}{|c|}{ At completion of follow up (visit 3 ) } \\
\hline \multicolumn{9}{|c|}{ Psychological distress: } \\
\hline HADS anxiety & 62 & $6.6(4.0)$ & $5.9(3.8)$ & 56 & $8.1(4.0)$ & $6.7(4.1)$ & $0.00(P=0.969)$ & $0.02(-1.21$ to 1.25$)$ \\
\hline HADS depression & 62 & $4.2(3.6)$ & $3.8(3.5)$ & 56 & $4.2(3.3)$ & $4.3(3.6)$ & $1.08(\mathrm{P}=0.300)$ & $0.4(-1.3$ to 0.39$)$ \\
\hline CIS total score & 67 & $7.0(6.9)$ & $7.3(8.1)$ & 62 & $8.6(8.2)$ & $7.6(8.8)$ & $0.64(P=0.424)$ & 0.89 (-1.3 to 3.06$)$ \\
\hline \multicolumn{9}{|l|}{ Voice: } \\
\hline Buffalo overall rating ${ }^{*}$ & 70 & $2.9(1.1)$ & $2.2(1.0)$ & 63 & $2.8(1.0)$ & $3.0(1.0)$ & $26.50(\mathrm{P}<0.001)$ & $0.82(0.5$ to 1.13$)$ \\
\hline Pitch perturbation $\dagger$ & 65 & $3.1(2.4)$ & $2.7(1.5)$ & 54 & $2.4(1.4)$ & $2.2(1.5)$ & $1.58(\mathrm{P}=0.212)$ & $0.35(-0.20$ to 0.89$)$ \\
\hline Amplitude perturbation $\dagger$ & 65 & $5.8(3.9)$ & $4.1(3.0)$ & 54 & $5.1(3.2)$ & $4.5(3.6)$ & $0.90(P=0.345)$ & $0.57(-0.62$ to 1.77$)$ \\
\hline Score on VPQ & 61 & $28.8(8.2)$ & $21.6(6.3)$ & 57 & $28.0(7.5)$ & $25.4(8.5)$ & $10.96(P=0.001)$ & 4.14 (1.67 to 6.61$)$ \\
\hline
\end{tabular}

HADS=hospital anxiety and depression scale; CIS=clinical interview schedule; VPQ=vocal performance questionnaire.

"Buffalo voice profile analysis has an "overall rating" as 1 of 10 items scored 0-5.

†The higher the score the more dysphonic the voice.

†Maximum score=60; the higher the score the more dysphonic the voice.

Table 3 Mean (SD) scores for quality of life (SF-36) variables at each visit for treatment and no treatment groups (patients with complete data). Higher scores indicate better health

\begin{tabular}{|c|c|c|c|c|c|c|c|c|}
\hline & \multicolumn{3}{|c|}{ Treatment } & \multicolumn{3}{|c|}{ No treatment } & \multirow[b]{2}{*}{$F$ value } & \multirow[b]{2}{*}{$\begin{array}{l}\text { Treatment effect } \\
\text { (points }(95 \% \mathrm{CI}) \text { ) }\end{array}$} \\
\hline & $\begin{array}{c}\text { No of } \\
\text { patients }\end{array}$ & $\begin{array}{c}\text { Visit } 1 \\
\text { (baseline) }\end{array}$ & Visit 2 or 3 & $\begin{array}{c}\text { No of } \\
\text { patients }\end{array}$ & $\begin{array}{c}\text { Visit } 1 \\
\text { (baseline) }\end{array}$ & Visit 2 or 3 & & \\
\hline \multicolumn{9}{|c|}{ At completion of treatment (visit 2) } \\
\hline Physical functioning & 63 & $66.7(32.6)$ & $66.6(34.0)$ & 65 & $64.3(31.0)$ & $64.9(30.3)$ & $0.01(P=0.92)$ & $0.33(-6.25$ to 6.90$)$ \\
\hline Social functioning & 63 & $78.0(25.7)$ & $81.1(26.4)$ & 62 & $70.7(28.5)$ & $73.9(27.6)$ & $0.45(P=0.50)$ & $2.5(-4.89$ to 9.88$)$ \\
\hline Role (physical) & 63 & $63.1(43.7)$ & $59.1(45.0)$ & 62 & $54.0(40.8)$ & $47.9(42.1)$ & $0.79(P=0.38)$ & $5.6(-6.94$ to 18.22$)$ \\
\hline Role (emotional) & 63 & $78.3(38.4)$ & $72.2(41.0)$ & 63 & $62.7(42.4)$ & $64.0(42.9)$ & $0.03(P=0.87)$ & $1.0(-11.44$ to 13.45$)$ \\
\hline Mental health & 63 & $71.5(18.1)$ & $76.9(18.3)$ & 63 & $65.1(19.5)$ & $66.1(20.1)$ & $6.08(P=0.02)$ & 6.63 (1.31 to 11.95$)$ \\
\hline Energy/fatigue & 63 & $55.3(20.7)$ & $59.8(21.4)$ & 63 & $48.6(22.2)$ & $50.8(23.3)$ & $2.03(P=0.16)$ & $3.95(-1.54$ to 9.44$)$ \\
\hline Pain & 62 & $64.1(28.8)$ & $69.0(28.5)$ & 60 & $59.7(23.1)$ & $62.0(30.6)$ & $0.87(P=0.35)$ & $3.96(-4.44$ to 12.36$)$ \\
\hline Health perception & 58 & $64.9(23.4)$ & $66.5(25.5)$ & 63 & $57.7(23.1)$ & $59.9(23.5)$ & $0.01(P=0.93)$ & $0.24(-4.76$ to 5.24$)$ \\
\hline \multicolumn{9}{|c|}{ At completion of follow up (visit 3 ) } \\
\hline Physical functioning & 59 & $67.7(31.8)$ & $69.1(30.2)$ & 56 & $64.6(30.5)$ & $64.1(30.4)$ & $0.55(P=0.46)$ & $2.5(-4.19$ to 9.19$)$ \\
\hline Social functioning & 58 & $77.4(25.9)$ & $78.2(26.1)$ & 55 & $74.0(25.1)$ & $72.9(28.4)$ & $0.55(P=0.46)$ & $3.11(-5.18$ to 11.4$)$ \\
\hline Role (physical) & 60 & $62.5(44.5)$ & $61.7(43.5)$ & 55 & $50.5(40.7)$ & $51.4(44.5)$ & $0.18(P=0.67)$ & $2.86(-10.34$ to 16.06$)$ \\
\hline Role (emotional) & 60 & $76.7(39.5)$ & $70.8(40.1)$ & 56 & $57.5(42.9)$ & $58.3(45.5)$ & $0.03(P=0.87)$ & $1.07(-12.26$ to 14.41$)$ \\
\hline Mental health & 58 & $70.8(18.6)$ & $74.7(17.8)$ & 55 & $65.2(19.8)$ & $67.9(19.7)$ & $1.58(\mathrm{P}=0.21)$ & $3.66(-2.12$ to 9.44$)$ \\
\hline Energy/fatigue & 58 & $54.6(20.4)$ & $56.1(24.6)$ & 55 & $49.5(21.2)$ & $51.0(23.6)$ & $0.07(P=0.80)$ & $0.81(-5.45$ to 7.06$)$ \\
\hline Pain & 56 & $62.2(28.5)$ & $60.7(29.0)$ & 56 & $61.3(23.9)$ & $55.8(28.7)$ & $1.04(\mathrm{P}=0.80)$ & $4.29(-4.03$ to 12.6$)$ \\
\hline Health perception & 55 & $63.2(23.7)$ & $65.0(25.8)$ & 55 & $58.4(24.0)$ & $59.3(26.0)$ & $0.28(P=0.60)$ & $1.46(-4.02$ to 6.94$)$ \\
\hline
\end{tabular}

points (effect size $0.76 \mathrm{SD}$ ). In conventional statistical terminology these are medium to large effects. Voice therapy had an effect on only one quality of life outcome variable-mental health. This was significantly better in the treatment group at completion of treatment but not at completion of follow up.

To address the issue of dropout we re-ran the analyses including all patients with data at baseline. For patients with missing data at visit 2 or visit 3 we entered the baseline values. This makes the conservative assumption that there was no difference in outcome between patients who dropped out from the treatment and no treatment groups. On reanalyses of the sensitivity scores, treatment effects (points), and confidence intervals for each of the outcome measures, the results retained their significant $P$ values and treatment effects (tables 4 and 5).

\section{Discussion}

This first randomised controlled trial of the efficacy of voice therapy for dysphonia has shown voice therapy to be effective in improving self rated and expert rated quality of voice. The magnitude of the observed mean improvements reflects clinically meaningful improvements in voice quality. The minimal change in laryngoscopic appearances during the study reflects the fact that many of the patients referred for non-surgical 
Table 4 Mean (SD) scores for key voice and psychological variables at each visit for treatment and no treatment groups (all patients with baseline data)

\begin{tabular}{|c|c|c|c|c|c|c|c|c|}
\hline \multirow[b]{2}{*}{ Test or questionnaire } & \multicolumn{3}{|c|}{ Treatment } & \multicolumn{3}{|c|}{ No treatment } & \multirow[b]{2}{*}{$\begin{array}{c}\text { Sensitivity score } \\
\text { (F value) }\end{array}$} & \multirow[b]{2}{*}{$\begin{array}{l}\text { Treatment effect } \\
\text { (points }(95 \% \mathrm{CI}) \text { ) }\end{array}$} \\
\hline & $\begin{array}{l}\text { No of } \\
\text { patients }\end{array}$ & $\begin{array}{c}\text { Visit 1 } \\
\text { (baseline) }\end{array}$ & $\begin{array}{c}\text { Visit } 2 \text { or } \\
3\end{array}$ & $\begin{array}{c}\text { No of } \\
\text { patients }\end{array}$ & $\begin{array}{c}\text { Visit } 1 \\
\text { (baseline) }\end{array}$ & Visit 2 or 3 & & \\
\hline \multicolumn{9}{|c|}{ At completion of treatment (visit 2) } \\
\hline \multicolumn{9}{|l|}{ Psychological distress: } \\
\hline HADS anxiety & 85 & $6.5(4.2)$ & $6.2(4.4)$ & 80 & $8.2(4.2)$ & $7.9(4.0)$ & $0.67(P=0.416)$ & $0.30(-0.43$ to 1.04 \\
\hline HADS depression & 85 & $4.1(3.5)$ & $4.0(3.7)$ & 80 & $4.6(3.6)$ & $4.5(3.4)$ & $0.30(P=0.584)$ & $0.17(-0.46$ to 0.81$)$ \\
\hline CIS total score & 99 & $7.6(7.5)$ & & 103 & $9.2(9.3)$ & & & \\
\hline \multicolumn{9}{|l|}{ Voice: } \\
\hline Buffalo overall rating ${ }^{\star}$ & 100 & $2.9(1.0)$ & $2.4(1.0)$ & 103 & $3.0(1.0)$ & $2.7(1.0)$ & $5.0(P=0.03)$ & 0.25 (0.003 to 0.47$)$ \\
\hline Pitch perturbation $†$ & 97 & $3.1(2.2)$ & $2.8(1.6)$ & 92 & $2.9(2.2)$ & $3.1(2.2)$ & $5.7(P=0.018)$ & $0.46(0.008$ to 0.84$)$ \\
\hline Amplitude perturbation $\uparrow$ & 97 & $6.1(4.3)$ & $5.1(3.5)$ & 92 & $6.1(5.3)$ & $6.5(5.5)$ & $11.9(P=0.001)$ & 1.5 (0.64 to 2.3$)$ \\
\hline VPQ total & 85 & $28.4(9.0)$ & $24.2(8.3)$ & 81 & $29.4(8.9)$ & $26.9(9.5)$ & $3.9(\mathrm{P}=0.051)$ & $1.9(-0.001$ to 3.8 \\
\hline \multicolumn{9}{|c|}{ At completion of follow up (visit 3) } \\
\hline \multicolumn{9}{|c|}{ Psychological distress: } \\
\hline HADS anxiety & 85 & $6.5(4.2)$ & $6.0(3.9)$ & 80 & $8.2(4.2)$ & $7.2(4.3)$ & $0.018(P=0.893)$ & $0.006(-0.88$ to 1.01 \\
\hline HADS depression & 85 & $4.1(3.5)$ & $3.8(3.4)$ & 80 & $4.6(3.6)$ & $4.6(3.8)$ & $1.51(P=0.221)$ & $0.37(-0.23$ to 0.98 \\
\hline CIS total score & 99 & $7.6(7.5)$ & $7.6(8.2)$ & 103 & $9.2(9.3)$ & $8.6(9.7)$ & $0.63(P=0.428)$ & $0.58(-2.01$ to 0.86 \\
\hline \multicolumn{9}{|l|}{ Voice: } \\
\hline Buffalo overall rating* & 100 & $2.9(1.0)$ & $2.4(1.0)$ & 103 & $3.0(1.0)$ & $3.1(1.0)$ & $29.80(P=0.000)$ & $0.62(0.40$ to 0.85$)$ \\
\hline Pitch perturbation $†$ & 97 & $3.1(2.2)$ & $2.8(1.6)$ & 92 & $2.9(2.2)$ & $2.8(2.3)$ & $0.140(\mathrm{P}=0.709)$ & $0.008(-0.35$ to 0.51$)$ \\
\hline Amplitude perturbation $\dagger$ & 97 & $6.1(4.3)$ & $5.0(4.0)$ & 92 & $6.1(5.3)$ & $5.8(5.6)$ & $2.8(P=0.096)$ & $0.80(-0.14$ to 1.7$)$ \\
\hline Score on VPQ $\ddagger$ & 85 & $28.4(9.0)$ & $23.3(8.3)$ & 81 & $29.4(8.9)$ & $27.7(9.9)$ & $12.32(P=0.001)$ & 3.69 (1.61 to 5.76$)$ \\
\hline
\end{tabular}

HADS=hospital anxiety and depression scale; $C I S=c$ linical interview schedule; $V P Q=v o c a l$ performance questionnaire.

"Buffalo voice profile analysis has an "overall rating" as 1 of 10 items scored $0-5$

the higher the score the more dysphonic the voice.

IMaximum score=60; the higher the score the more dysphonic the voice.

Table 5 Mean (SD) scores for quality of life (SF-36) variables at each visit for treatment and no treatment groups (all patients with baseline data). Higher scores indicate better health

\begin{tabular}{|c|c|c|c|c|c|c|c|c|}
\hline & \multicolumn{3}{|c|}{ Treatment } & \multicolumn{3}{|c|}{ No treatment } & \multirow[b]{2}{*}{ F value } & \multirow[b]{2}{*}{$\begin{array}{l}\text { Treatment effect } \\
\text { (points }(95 \% \mathrm{CI}) \text { ) }\end{array}$} \\
\hline & $\begin{array}{c}\text { No of } \\
\text { patients }\end{array}$ & $\begin{array}{c}\text { Visit } 1 \\
\text { (baseline) }\end{array}$ & Visit 2 or 3 & $\begin{array}{l}\text { No of } \\
\text { patients }\end{array}$ & $\begin{array}{c}\text { Visit } 1 \\
\text { (baseline) }\end{array}$ & Visit 2 or 3 & & \\
\hline \multicolumn{9}{|c|}{ At completion of treatment (visit 2) } \\
\hline Physical functioning & 81 & $65.3(33.1)$ & $65.2(34.2)$ & 81 & $64.7(30.5)$ & $65.2(29.9)$ & $0.040(P=0.841)$ & $0.53(-4.69$ to 5.75$)$ \\
\hline Social functioning & 82 & $74.4(26.7)$ & $76.9(27.6)$ & 79 & $70.2(28.6)$ & $72.8(27.9)$ & $0.101(P=0.751)$ & 0.94 (-6.81 to 4.92$)$ \\
\hline Role (physical) & 82 & $59.8(43.7)$ & $56.7(44.5)$ & 80 & $53.8(41.5)$ & $49.1(42.6)$ & $0.473(P=0.493)$ & $3.44(-13.33$ to 6.45 \\
\hline Role (emotional) & 82 & $70.7(42.4)$ & $66.0(43.4)$ & 81 & $61.1(43.3)$ & $62.9(43.4)$ & $0.389(P=0.534)$ & $3.10(-6.71$ to 12.90 \\
\hline Mental health & 82 & $69.9(19.2)$ & $74.0(19.8)$ & 79 & $64.3(19.3)$ & $65.1(19.8)$ & $4.784(\mathrm{P}=0.030)$ & $4.74(-9.01$ to -0.46 \\
\hline Energy/fatigue & 82 & $53.2(21.0)$ & $56.7(22.0)$ & 79 & $47.2(21.7)$ & $48.9(22.8)$ & $1.611(P=0.206)$ & $2.81(-7.17$ to 1.56$)$ \\
\hline Pain & 80 & $62.6(28.9)$ & $66.4(28.9)$ & 79 & $58.4(25.6)$ & $60.2(31.0)$ & $0.785(\mathrm{P}=0.377)$ & $2.94(-9.48$ to 3.61$)$ \\
\hline Health perception & 78 & $62.2(23.6)$ & $63.4(25.4)$ & 80 & $56.7(23.4)$ & $58.5(23.8)$ & $0.002(P=0.963)$ & $0.009(-3.76$ to 3.94$)$ \\
\hline \multicolumn{9}{|c|}{ At completion of follow up (visit 3) } \\
\hline Physical functioning & 81 & $65.3(33.1)$ & $66.2(32.1)$ & 81 & $64.7(30.5)$ & $64.4(30.5)$ & $0.323(P=0.570)$ & $1.39(-6.21$ to 3.43$)$ \\
\hline Social functioning & 82 & $74.4(26.7)$ & $75.0(26.9)$ & 79 & $70.2(28.6)$ & $69.5(30.6)$ & $0.533(P=0.466)$ & $2.22(-8.21$ to 3.78$)$ \\
\hline Role (physical) & 82 & $59.8(43.7)$ & $59.1(42.9)$ & 80 & $53.8(41.5)$ & $54.4(44.1)$ & $0.007(P=0.934)$ & $0.40(-9.90$ to 9.11$)$ \\
\hline Role (emotional) & 82 & $70.7(42.4)$ & $66.5(42.3)$ & 81 & $61.1(43.3)$ & $61.7(45.1)$ & $0.216(P=0.643)$ & $2.25(-7.31$ to 11.81 \\
\hline Mental health & 82 & $69.9(19.2)$ & $72.7(18.9)$ & 79 & $64.3(19.3)$ & $66.1(19.4)$ & $1.45(\mathrm{P}=0.230)$ & 2.59 (-6.83 to 1.66$)$ \\
\hline Energy/fatigue & 82 & $53.2(21.0)$ & $54.3(24.0)$ & 79 & $47.2(21.7)$ & $48.2(23.5)$ & $0.079(P=0.780)$ & $0.63(-5.03$ to 3.78$)$ \\
\hline Pain & 80 & $62.6(28.9)$ & $61.5(29.3)$ & 79 & $58.4(25.6)$ & $54.6(28.5)$ & $1.45(\mathrm{P}=0.230)$ & $3.60(-9.51$ to 2.31$)$ \\
\hline Health perception & 78 & $62.2(23.6)$ & $63.5(24.2)$ & 80 & $56.7(23.4)$ & $57.3(24.9)$ & $0.315(P=0.576)$ & 1.08 (-4.90 to 2.73$)$ \\
\hline
\end{tabular}

voice therapy have, by definition, relatively normal laryngeal appearances.

Psychological distress was not significantly reduced as a result of treatment. Voice therapy had a significant effect on one quality of life variable-mental health-at the end of treatment, but this was not maintained at follow up. A subgroup of patients remain psychologically distressed despite receiving treatment. Speech and language therapists often use psychological strategies but often acquire psychological training after qualification and in what has been described as an ad hoc manner. ${ }^{17}$ If patients with high psychological distress could be identified by screening they could be referred for psychological intervention, perhaps from a clinical psychologist. ${ }^{18}$

The disconcertingly abnormal SF-36 results highlight the importance of effective vocal communication for an individual's psychosocial wellbeing. Indeed, the level of psychological morbidity may also mainly reflect the greatly reduced quality of life in patients with dysphonia. Such interrelations underline the importance of a holistic treatment for reduction in symptoms and improvement in overall functioning.

In conclusion, this study shows that voice therapy is effective in improving self rated and observer rated measures of voice quality. However, voice therapy does 


\section{What is already known on this topic}

Many patients with dysphonia are treated by voice therapy

The effectiveness of voice therapy in a diverse group of patients is unknown

\section{What this study adds}

Voice therapy is an effective treatment for dysphonia in terms of report by patients and perceptual ratings by an expert

Psychological distress and reduction in general health status are common in patients with dysphonia but are not significantly affected by a course of voice therapy

not significantly affect laryngeal pathophysiology or reduce the high levels of psychological distress that characterise patients with dysphonia.

We acknowledge the contributions of Nicola Bradshaw and Shonagh Scott, who were both involved as research assistants in the collection of data for this paper; Dr Martha Whiteman for running the sensitivity analyses; Catherine Dunnet, chief speech and language therapist at Glasgow Royal Infirmary, for contributions to the project and comments on a draft of the paper; and Professor Stuart Gatehouse for his comments on an earlier draft of the paper

Contributors: KMacK initiated and coordinated the formulation of the primary study hypothesis, discussed core ideas, designed the protocol, and participated in data collection and the writing of the paper. AM discussed core ideas, coordinated the study, carried out the psychological analysis, ran the statistical analyses of treatments, and was involved in writing the paper. JAW initiated and coordinated the formulation of the primary study hypothesis, discussed core ideas, designed the protocol, and contributed to writing the paper. CS discussed core ideas, designed the protocol, and carried out all the voice therapy. IJD initiated and coordinated the formulation of the primary study hypothesis, discussed core ideas, designed the protocol, supervised the statistical analyses, and was principally involved in writing the paper. The guarantor for the paper is KMacK.

Funding: Scottish Office Home and Health Department (grant reference: $\mathrm{K} / \mathrm{RED} / 4 / \mathrm{C} 249$ ).

Competing interests: None declared.

1 Wilson JA, Deary IJ, Scott S, MacKenzie K. Functional dysphonia [editorial]. $B M J$ J 1995;311:1039-40.

2 Scott S, Wilson JA, Robinson K, MacKenzie K. Patient reported problems associated with dysphonia. Clin Otolaryngol 1997;22:37-40.

3 Aronson A, Peterson H, Litin E. Psychiatric symptomology in functional dysphonia and aphonia.J Speech Hear Disord 1996;31:115-27.

4 Matas M. Psychogenic voice disorders: literature review and case report Can J Psychiatry 1991;36:363-5.

5 Gerritsma EJ. An investigation into some personality characteristics of patients with psychogenic aphonia and dysphonia. Folia Phomiatr Logop 1991;43:13-20.

6 House A, Andrews H. The psychiatric and social characteristics of patients with functional dysphonia. J Psychosom Res 1987;31:483-90.

7 House A, Andrews H. Life events and difficulties preceding the onset of functional dysphonia. J Psychosom Res 1988;32:311-19.

8 Deary IJ, Scott S, Wilson IM, White A, MacKenzie K, Wilson JA. Personality and psychological distress in dysphonia. $\mathrm{Br} J$ Health Psychol 1997;2:333-41.

9 White A, Deary IJ, Wilson JA. Psychiatric disturbance and personality traits in dysphonic patients. Eur J Disord Commun 1997;32:307-14.

10 Fairbanks G. Voice and articulation drillbook, 2nd ed. New York: Harper and Brothers, 1960.

11 Wilson DK. Voice problems of children, 3rd ed. Baltimore: Williams and Wilkins, 1987

12 Carding PN, Horsley IA. An evaluation of voice therapy in non-organic dysphonia. Eur J Disord Commun 1992;27:137-48.

13 Zigmond AS, Snaith RP. The hospital anxiety and depression scale. Acta Psychiatr Scand 1983; 67:361-70.

14 Lewis G, Pelosi AJ, Araya R, Dunn G. Measuring psychiatric disorder in the community: a standardised assessment for use by lay interviewers Psychol Med 1992;22:465-86.

15 Jenkinson C, Coulter A, Wright L. Short form 36 (SF36) health survey questionnaire: normative data for adults of working age. BMJ 1993;306:1437-40.

16 Dunnet CP, MacKenzie K, Robinson K, Sellars GC, Wilson JA. Voice therapy for dysphonia-still more art than science? Eur J Disord Commun 1997;32:333-43.

17 Elias A, Raven R, Butcher P, Littlejohns D. Speech therapy for psychogenic voice disorder: a survey of current practice and training. $\mathrm{BrJ}$ Disord Commun 1989;24:61-76.

18 Scott S, Deary IJ, Wilson JA, MacKenzie K. Functional dysphonia-a role for psychologists? Psychol Health Med 1997;2:169-80.

(Accepted 8July 2001) 